\title{
ULTRAHIGH ENERGY NEUTRINOS
}

\author{
SHARADA IYER DUTTA \\ Department of Physics and Astronomy, \\ State University of New York at Stony Brook \\ Stony Brook, New York 11794, USA \\ MARY HALL RENO* \\ Department of Physics and Astronomy, University of Iowa \\ Iowa City, Iowa 52242, USA \\ INA SARCEVIC \\ Department of Physics, University of Arizona \\ Tucson, Arizona 85721, USA
}

\begin{abstract}
The ultrahigh energy neutrino cross section is well understood in the standard model for neutrino energies up to $10^{12} \mathrm{GeV}$. Tests of neutrino oscillations $\left(\nu_{\mu} \leftrightarrow \nu_{\tau}\right)$ from extragalactic sources of neutrinos are possible with large underground detectors. Measurements of horizontal air shower event rates at neutrino energies above $10^{10} \mathrm{GeV}$ will be able to constrain nonstandard model contributions to the neutrino-nucleon cross section, e.g., from mini-black hole production.
\end{abstract}

\section{INTRODUCTION}

The existence of ultrahigh energy (UHE) cosmic rays comprised of nucleons guarantees the presence of ultrahigh energy neutrinos. Over megaparsec distances, UHE nucleons interact with the $2.7 \mathrm{~K}$ microwave background radiation to produce the delta resonance, which decays to a pion and a nucleon. Charged pions decay to neutrinos and muons, followed by muon decay, leading to fluxes of electron and muon neutrinos. Beginning with the pioneering work of Ref. 1, and continuing to recent improvements [2, 3], there are quantitative predictions for fluxes of neutrinos with $E_{\nu} \sim 10^{8} \mathrm{GeV}$ and higher energies.

At somewhat lower energies, one expects that sources of $\mathrm{TeV}$ photons such as active galactic nuclei should also produce neutrinos. The attenuation length of the photon effectively cuts off the photon energy at $E_{\gamma} \sim 1 \mathrm{TeV}$ for sources farther than $100 \mathrm{Mpc}$ from the Earth. The long interaction length of neutrinos means that neutrino attenuation from distant sources is not an issue over astronomical distances due to low densities.

A common feature to neutrino flux models is that roughly two muon neutrinos/antineutrinos appear for each electron neutrino/antineutrino at the production site. (In the following discussion, we do not distinguish between $\nu$ and $\bar{\nu}$.) In the past few years, the $\mathrm{Su}-$ perKamiokande experiment [4] together with the SNO 5 results point to physics beyond the standard model: neutrino masses and mixing. Parameters in the mixing matrix lead fairly robustly to an equal distribution of fluxes

\footnotetext{
*Presented at Neutrinos and Implications for Physics Beyond the
} Standard Model, Stony Brook, NY, October 11-13, 2002. of neutrinos between the three neutrino flavors $[\underline{6}]$ :

$$
F_{\nu_{e}}=F_{\nu_{\mu}}=F_{\nu_{\tau}}
$$

after propagating over astronomically large distances. UHE tau neutrino interactions with nucleons in the Earth and atmosphere may provide signals of this phenomenon. Tests for deviations from the standard model ultrahigh energy cross section are another probe of non-standard model physics with UHE neutrinos.

The neutrino energy regime determines the detection method. For $E_{\nu} \leq 10^{6} \mathrm{GeV}$, underground detectors can be used to record upward muons and electromagnetic/hadronic showers. Experiments include water or ice Cherenkov detectors like AMANDA []], NESTOR [8], ANTARES [9] and IceCube 10]. Attenuation of the neutrino flux in Earth is important, especially the difference between $\nu_{\mu}$ and $\nu_{\tau}$ attenuation $11,12,13$. In the energy range above $10^{8}-10^{9} \mathrm{GeV}$, detection of horizontal air showers and radio detection are used to determine the UHE $\nu$ fluxes and to probe the neutrino cross section 14, 15, 16, 17, 18, 19]. At intermediate energies, the double-bang signal of both production and decay vertices of the $\nu_{\tau} \rightarrow \tau \rightarrow \nu_{\tau} X$ process can be used to identify $\nu_{\tau} \mathrm{S} 20$. Other detection possibilities include using the Earth as a tau neutrino converter, then searching for the shower from the decay of the emerging tau 21].

We present results in two of these examples: upward neutrino fluxes and their detection, and the potential for limits on the non-standard neutrino cross section from the OWL 16 proposed detector. In this latter case, the non-standard model considered is mini-black hole production and decay 22, 23, 24, 25, 26, 27, 28], a feature of models with $\mathrm{TeV}$-scale compactification of extra dimensions [29]. Before going into detail of these two examples, we review the theoretical status of the neutrino cross section at high energies. 


\section{UHE NEUTRINO CROSS SECTION}

The neutrino-nucleon cross section is straightforward to calculate in the standard model 30$]$. The charged current cross section is

$$
\frac{d^{2} \sigma}{d x d Q^{2}}=\frac{G_{F}^{2}}{\pi}\left(\frac{M_{W}^{2}}{Q^{2}+M_{W}^{2}}\right)^{2} \cdot\left[q(x, Q)+(1-y)^{2} \bar{q}(x, Q)\right]
$$

for $W$ boson momentum squared $q^{2}=-Q^{2}$, lepton energy difference $q^{0}=E-E^{\prime}=\nu$, Bjorken $x=Q^{2} /(2 M \nu)$ and $y=\nu / E$ with nucleon mass $M$ and $W$ mass $M_{W}$. The parton distribution function combinations $q(x, Q)$ and $\bar{q}(x, Q)$ are detailed in, for example, Ref. 31. The cross section depends crucially on the parton distribution functions [32]. As $\ln \left(Q^{2}\right)$ gets large, the parton distributions at small- $x$ increase, however, the $W$ boson propagator decreases with increasing $Q^{2}$. At sufficiently high energies, the value of $Q^{2}$ saturates at $Q^{2} \sim M_{W}^{2}$, so the relevant values of $x$ for a given incident neutrino energy $E$ is approximately

$$
x \sim \frac{M_{W}^{2}}{2 M E} .
$$

This translates to $x \sim 10^{-2}$ for $E \sim 10^{6} \mathrm{GeV}$, and $x \sim$ $10^{-8}$ for $E \sim 10^{12} \mathrm{GeV}$.

Measurements of the parton distribution functions have been made down to $x \sim 10^{-6}$ at $Q^{2} \sim 0.1 \mathrm{GeV}^{2}$ at HERA 33]. At a more relevant $Q^{2} \sim M_{W}^{2}$, parton distributions have been measured to $x \sim 10^{-3}[34]$.

Beyond the measured regime, one is guided by theoretical arguments concerning the small- $x$ parton distribution functions and their QCD evolution. As discussed in detail in Ref. 35, for $x g\left(x, Q_{0}\right) \sim x^{-\lambda}$ at $x \ll 1$, then $x g(x, Q) \sim x^{-\lambda}$ for $\lambda \gtrsim 0.3$. At small values of $x$, the quark distribution functions are dominated by the quark sea, which come from $g \rightarrow q \bar{q}$, so the sea distributions have the same power law dependence: $x q(x, Q) \sim x^{-\lambda}$.

Modern parton distribution function fits have $\lambda \sim$ $0.3-0.4$, and extrapolations to small- $x$ by this power law agree well with perturbative QCD evolution, as demonstrated, for example, by Glück, Kretzer and Reya in Ref. 36. Kwiecinski, Martin and Stasto have used a BFKLtype evolution at small- $x$ and obtain cross sections that are in agreement with power law extrapolations 37 .

At very small values of $x$, recombination processes such as $g g \rightarrow g$ should become important, ultimately leading to a flattening of the neutrino cross section as a function of energy. The recombination regime is unimportant for neutrino energies up to $10^{12} \mathrm{GeV}$, though it should become important at even higher energies [38].

Fig. 1 shows the neutrino interaction length, proportional to the inverse of the cross section:

$$
\mathcal{L}_{i n t}=\frac{1}{N_{A} \sigma_{\nu N}}
$$

with $N_{A}$ equal to Avogadro's number. For neutrino energies above a few tens of TeV, the Earth's diameter is

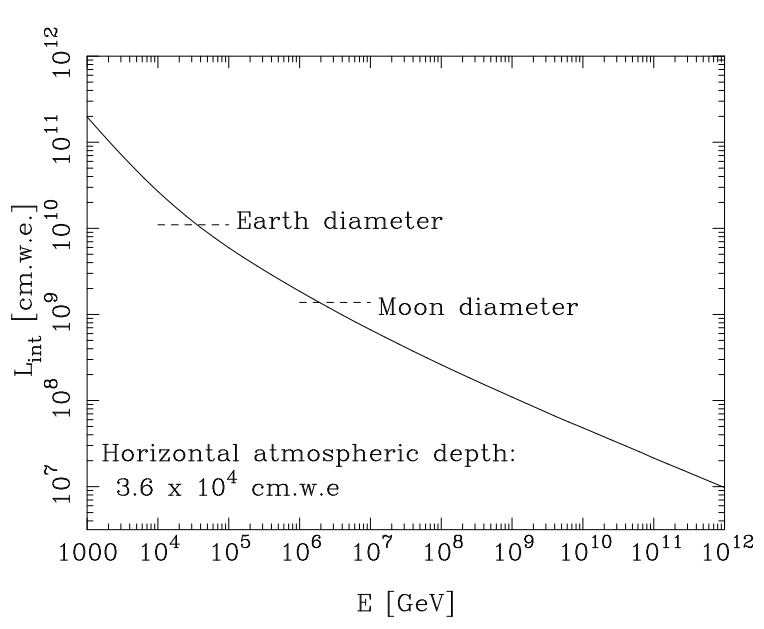

FIG. 1: The standard model neutrino interaction length in cm.w.e. $=\mathrm{g} / \mathrm{cm}^{2}$, with reference distances through the Earth and the Moon.

larger than the attenuation length. Even for neutrinos with $E=10^{12} \mathrm{GeV}$, the horizontal atmospheric depth is short compared to the neutrino interaction length.

\section{UPWARD NEUTRINOS IN UNDERGROUND DETECTORS}

In our discussion of upward neutrinos, we restrict our attention to neutrino energies below $10^{6} \mathrm{GeV}[12]$. For the upward neutrino flux, depending on the nadir angle, attenuation due to neutrino passage through the Earth is important. Neutrino oscillations of $\nu_{\mu} \leftrightarrow \nu_{\tau}$ lead to the arrival of equal fluxes of $\nu_{\mu}$ and $\nu_{\tau}$ at the surface of the Earth, however, it has been noted that the attenuation of the two species is quite different due to the short lifetime of the $\tau[11,12,13]$.

Muon and tau neutrinos have the same neutral current and charged current cross sections above $1 \mathrm{TeV}$. (At 1 $\mathrm{TeV}$, the charged current cross section for $\nu_{\tau} \rightarrow \tau$ is about $5 \%$ lower than the $\nu_{\mu}$ charged current $(\mathrm{CC})$ cross section due to the $\tau$ mass. At $100 \mathrm{GeV}$, the $\nu_{\tau} \mathrm{CC}$ cross section is about $25 \%$ smaller [39].) The difference in the attenuation of $\nu_{\mu}$ and $\nu_{\tau}$ fluxes has to do with the fact that muons lose energy as they traverse a medium before they decay. Electromagnetic energy loss for muons is significant, so the neutrinos from muon decay have much lower energies than the parent muon energy. On the other hand, electromagnetic energy loss for $\tau$ 's is important only for tau energies above $\sim 10^{8} \mathrm{GeV}[40]$. The muon neutrino and tau neutrino transport equations are

$$
\begin{aligned}
& \frac{\partial F_{\nu_{\mu}}(E, X)}{\partial X}=-\frac{F_{\nu_{\mu}}(E, X)}{\mathcal{L}_{\nu}^{\text {int }}(E)} \\
& \quad+\int_{E}^{\infty} d E_{y} G^{\nu_{\mu} \rightarrow \nu_{\mu}}\left(E, E_{y}, X\right)
\end{aligned}
$$




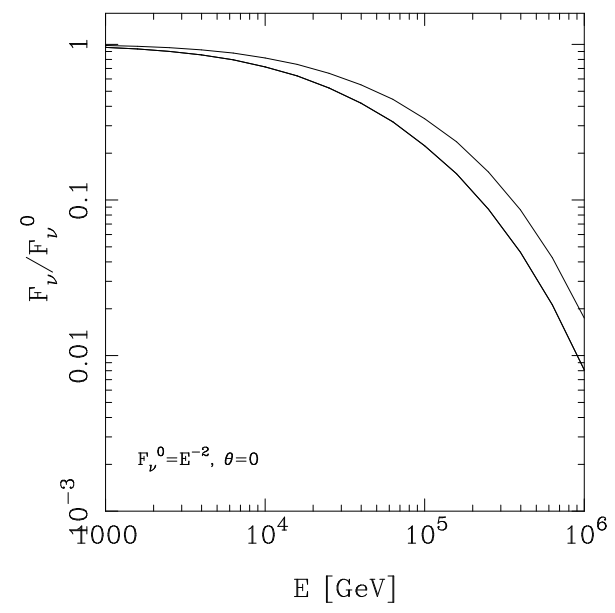

FIG. 2: The ratio of the attenuated flux to incident flux for $\nu_{\tau}$ (upper curve) and $\nu_{\mu}$ (lower curve) for $F_{\nu}^{0} \sim E^{-2}$ at nadir angle 0.

$$
\begin{aligned}
& \frac{\partial F_{\nu_{\tau}}(E, X)}{\partial X}=-\frac{F_{\nu_{\tau}}(E, X)}{\mathcal{L}_{\nu}^{\text {int }}(E)} \\
& +\int_{E}^{\infty} d E_{y}\left[G^{\nu_{\tau} \rightarrow \nu_{\tau}}\left(E, E_{y}, X\right)+G^{\tau \rightarrow \nu_{\tau}}\left(E, E_{y}, X\right)\right] \\
& \frac{\partial F_{\tau}(E, X)}{\partial X} \simeq-\frac{F_{\tau}(E, X)}{\mathcal{L}_{\tau}^{\text {dec }}} \\
& \quad+\int_{E}^{\infty} d E_{y} G^{\nu_{\tau} \rightarrow \tau}\left(E, E_{y}, X\right)
\end{aligned}
$$

where, for example,

$$
G^{\nu_{\tau} \rightarrow \nu_{\tau}}\left(E, E_{y}, X\right)=\left[\frac{F_{\nu_{\tau}}\left(E_{y}, X\right)}{\mathcal{L}_{\nu}^{\text {int }}}\right] \frac{d n^{N C}}{d E}\left(E_{y}, E\right) .
$$

in terms of the neutrino flux $F_{\nu_{\tau}}$ and the cross section normalized neutral current energy distribution $d n^{N C} / d E$. We have made the approximation that tau energy loss is negligible, and that only the decay of the tau is relevant, neglecting tau CC interactions.

The feed-down of neutrinos from $\nu_{\tau} \rightarrow \tau$ is evident in comparison of the attenuated $\nu_{\tau}$ flux with the $\nu_{\mu}$ flux [12, 13]. In Figs. 2 and 3 we show the ratio of the attenuated flux to the incident flux for $\nu_{\tau}$ (upper curve) and $\nu_{\mu}$ (lower curve) at nadir angle 0 for two different fluxes, parameterized by

$$
F_{\nu}^{0}=E^{-\gamma-1} /\left(1+E / E_{0}\right)^{\alpha}
$$

for $E_{0}=10^{8} \mathrm{GeV}$. In Fig. 2, $\gamma=1$ and $\alpha=0$, and in Fig. $3, \gamma=0$ and $\alpha=2$. The tau neutrino pileup is more pronounced for the flatter fluxes. At larger nadir angles, the pileup reduces because the number of interactions reduces, but the attenuation is also less.

Experimentally, one would like to exploit the effect of the tau neutrino pile-up and to identify the tau neutrino component of the flux. In a manner similar to the lower energy comparison of the neutral current to

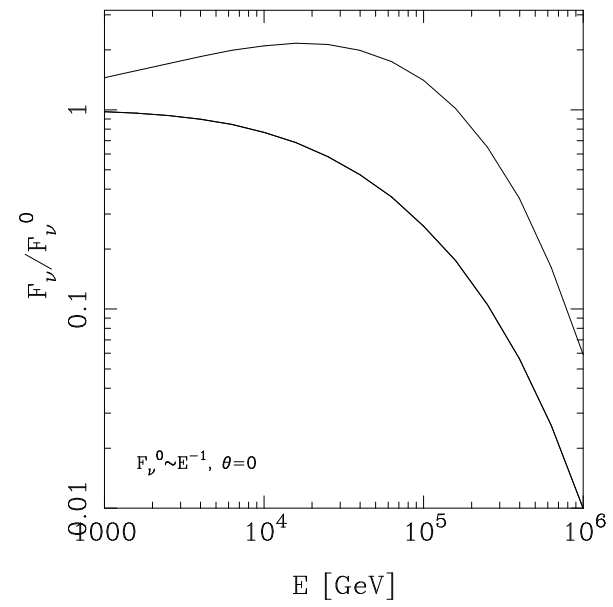

FIG. 3: The ratio of the attenuated flux to incident flux for $\nu_{\tau}$ (upper curve) and $\nu_{\mu}$ (lower curve) for $F_{\nu}^{0} \sim E^{-1}$ at nadir angle 0 .

charged current event rates $[\underline{5}$, for neutrinos with energies above $1 \mathrm{TeV}$, one can compare the upward electromagnetic/hadronic shower event rate from

$$
\begin{aligned}
& \nu_{\tau} N \rightarrow \tau+\text { hadrons, } \tau \rightarrow \nu_{\tau}+\text { hadrons } \\
& \nu_{\tau} N \rightarrow \tau+\text { hadrons }, \quad \tau \rightarrow \nu_{\tau}+e+\nu_{e} \\
& \nu_{\tau, \mu, e} N \rightarrow \nu_{\tau, \mu, e}+\text { hadrons } \\
& \nu_{e} N \rightarrow e+\text { hadrons }
\end{aligned}
$$

with the upward muon event rate from

$$
\begin{aligned}
& \nu_{\mu} N \rightarrow \mu+X \\
& \nu_{\tau} N \rightarrow \tau+X, \quad \tau \rightarrow \nu_{\tau}+\mu+\nu_{\mu} .
\end{aligned}
$$

We have made a detailed comparison in Ref. 12. By measuring the upward shower and muon event rates with an energy threshold of $10 \mathrm{TeV}$, one should be able to see the effect of the tau neutrino component of the incident neutrino flux. This is relatively independent of the a priori unknown spectrum of the incident flux, except for the overall normalization (so that there are enough events).

An interesting possibility for a class of input neutrino fluxes is the enhancement of the upward muon rate from $\nu_{\tau} \rightarrow \tau \rightarrow \nu_{\mu}$ where the final muon neutrino interacts to produce a muon [41]. The muon neutrinos from tau decays are called "secondary neutrinos." There is incremental energy loss at each of the processes: CC production of the tau, then muonic decay of the tau. This results in a contribution to the muon neutrino flux 42], as seen in Fig. 4 for $F \sim 1 / E$ for nadir angle 0 . The upper dashed line is $F_{\nu} / F_{\nu}^{0}$ for the $\nu_{\tau}$ flux, the lower dashed line is for $\nu_{\mu}$ excluding the secondary neutrinos, while the solid line is the secondary muon neutrino flux. The implications for the upward muon rate are shown in Fig. 5. The secondary neutrino flux for the $1 / E^{2}$ incident flux is relatively less important. For less steep fluxes than $1 / E$, one expects that the secondary flux will be enhanced. 


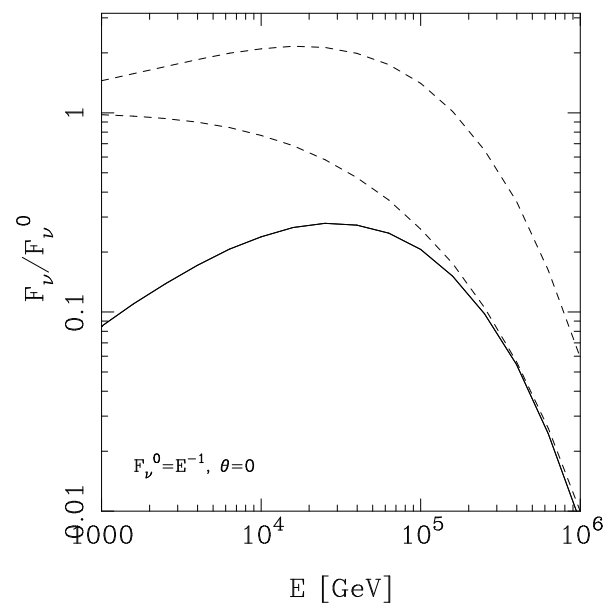

FIG. 4: The ratio of the attenuated flux to incident flux for $\nu_{\tau}$ (upper dashed curve) and $\nu_{\mu}$ (lower dashed curve) and secondary $\nu_{\mu}$ (solid curve) for $F_{\nu}^{0} \sim E^{-1}$ at nadir angle 0 .

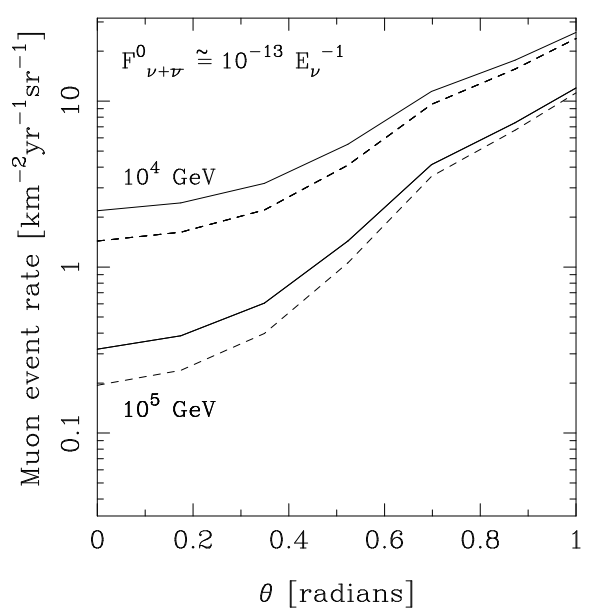

FIG. 5: Upward muon event rate for $F_{\nu}^{0} \sim 10^{-13}(\mathrm{GeV} / E)$ $\left(\mathrm{cm}^{2} \mathrm{~s} \mathrm{sr} \mathrm{GeV}\right)^{-1}$ without secondary neutrinos (dashed curve) and with secondary neutrinos (solid curve) for $E_{\mu} \geq 10^{4}, 10^{5}$ $\mathrm{GeV}$.

\section{HORIZONTAL AIR SHOWER SIGNALS OF MINI-BLACK HOLES}

As Fig. 1 indicates, the standard model neutrino interaction length is large compared to the horizontal atmospheric depth (zenith angle $90^{\circ}$ at sea level). Unlike strongly interacting particles, neutrinos can penetrate deep into the atmosphere. Horizontal air showers initiated deep in the atmosphere are a signal of neutrinos. Non-observation of an enhancement of horizontal air shower event rates limit a combination of the neutrinonucleon cross section and the neutrino flux [18, 19]. Using the cosmogenic neutrino flux from cosmic ray scattering with the $2.7 \mathrm{~K}$ photons, it is possible to set limits on nonstandard contributions to the cross section.

The cosmogenic neutrino flux, for the sum of electron

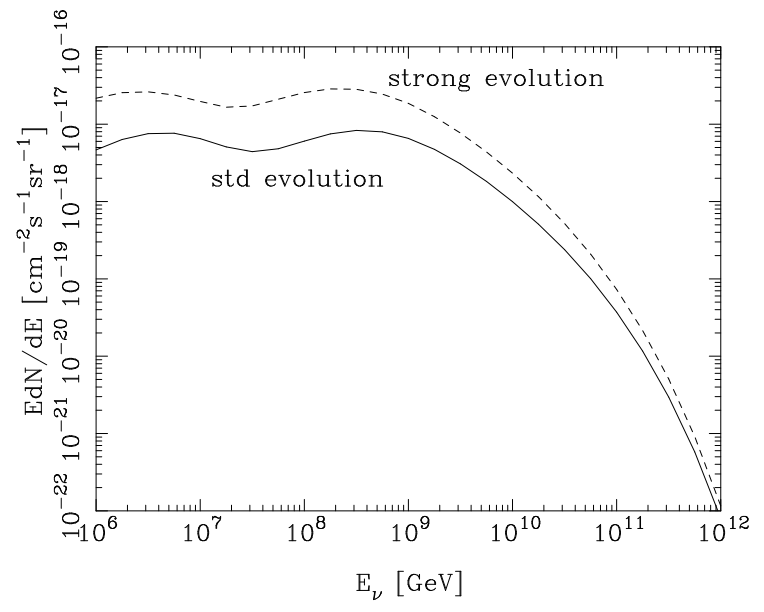

FIG. 6: The cosmogenic electron neutrino plus antineutrino flux of Engel, Seckel and Stanev. ${ }^{3}$

neutrino plus antineutrino, evaluated by Engel, Seckel and Stanev [3] (ESS) is shown in Fig. 6. Their results are shown for two models of source evolution: one parameterized like $(1+z)^{3}$ for redshift $z<1.9$ (standard evolution), and the other scaling like $(1+z)^{4}$ for the same redshift (strong evolution).

Discussed here are contributions from mini-black holes, predicted by models with the Planck scale in the $\mathrm{TeV}$ range and a number of extra dimensions 29], and the potential of an orbiting observatory to detect the enhanced rate of horizontal air showers [43]. There is an extensive literature on black hole production and detection by neutrino telescopes 19, 22, 23, 24, 25, 26, 27, 28]. It appears that enhancements of the cross section from $t$-channel Kaluza-Klein graviton exchange is not an important effect 23, 24].

Black hole production in these models is determined by the geometrical cross section, depending on the Schwarzschild radius. The Schwarzschild radius $r_{S}$, assuming $n$ compactified dimensions and Planck scale $M_{D}$, is given by [4]

$$
r_{S}=\frac{1}{M_{D}}\left[\frac{M_{\mathrm{BH}}}{M_{D}}\left(\frac{2^{n} \pi^{\frac{n-3}{2}} \Gamma\left(\frac{3+n}{2}\right)}{2+n}\right)\right]^{\frac{1}{1+n}}
$$

Here, $M_{\mathrm{BH}}$ is the mass of the black hole. The geometrical cross section for neutrino interaction with parton $j$ is then

$$
\hat{\sigma}(\nu j \rightarrow B H)=\pi r_{S}^{2}\left(M_{B H}=\sqrt{\hat{s}}\right) \theta\left(\sqrt{\hat{s}}-M_{B H}^{\min }\right)
$$

for $\hat{s}$ equal to the neutrino-parton center of mass energy. Eq. (10) enforces the requirement that $\hat{s}$ be larger than some minimum energy-squared, certainly larger than the Planck scale. Convoluting with the parton distribution functions $f_{i}$ after setting $\hat{s}=x s$ give a non-standard 


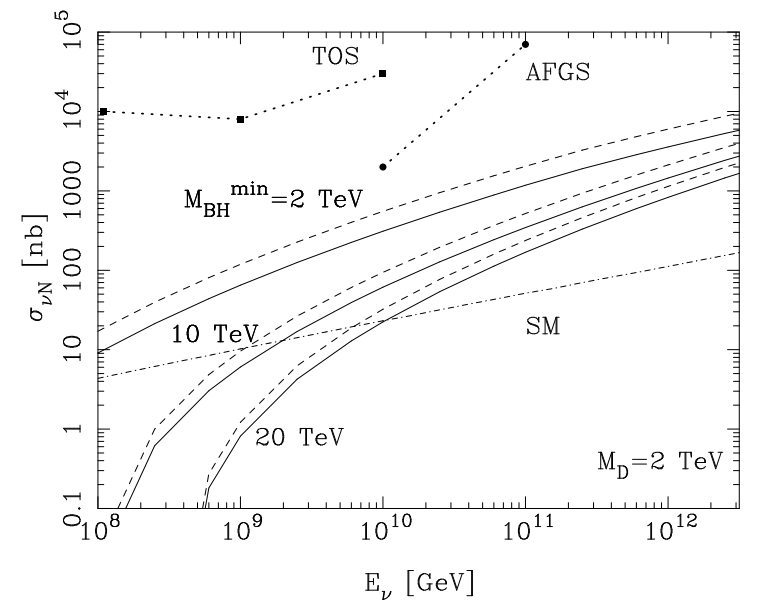

FIG. 7: The black hole production cross section for $n=4$ (solid) and $n=6$ (dashed) for several values of the minimum black hole mass, for a Planck scale of $M_{D}=2 \mathrm{TeV}$. The standard model cross section is also shown (SM) with the dot-dashed line. The limits of Tyler, Olinto and Sigl ${ }^{18}$ (TOS) and Anchordoqui et al. ${ }^{19}$ (AFGS) are shown with the dotted lines.

model contribution to the neutrino-nucleon cross section:

$$
\sigma(\nu N \rightarrow \mathrm{BH})=\sum_{i} \int_{\frac{\left(M_{B H}^{\min )^{2}}\right.}{s}}^{1} d x \hat{\sigma}_{i}^{B H}(x s) f_{i}\left(x, Q^{2}\right) .
$$

The black hole is expected to rapidly evaporate into standard model particles, and we assume that all of the energy of the black hole goes into the shower it produces 44].

Experimental constraints on deviations from Newtonian gravity exclude $n=1$ and $M_{D} \sim 1 \mathrm{TeV}[19]$. For $n=2, M_{D}>3.5 \mathrm{TeV}$ is consistent with tests of the inverse square law, [45] while for larger $n$, there are no constraints from tests of Newtonian gravity for $M_{D} \sim 1$ $\mathrm{TeV}$. Collider experiments put a lower limit on $M_{D}$ at approximately $1 \mathrm{TeV}$ [19, 46].

Fig. 7 shows a representative set of parameter choices for the number of extra dimensions $n$ and the minimum black hole mass, given a Planck scale of $M_{D}=2 \mathrm{TeV}$. Also shown are curves representing upper limits on the cross section from Tyler, Olinto and Sigl [18] (TOS) and Anchordoqui, Feng, Goldberg and Shapere 19] (AFGS), were they have used cosmogenic fluxes similar to that of ESS. These upper limits come from the AGASA and Fly's Eye limits on an excess of horizontal air shower events.

The largest enhancement of the cross section comes at the highest energies. Detectors optimized to this energy range will be able to put the most stringent limits on the cross section. The proposed Orbiting Wide-Angle LightCollectors Experiment 16! (OWL) and the Extreme Universe Space Observatory [17] (EUSO) are two such detectors. The OWL experiment would involve two satellites with photodetectors, orbiting $640 \mathrm{~km}$ above the Earth. The EUSO experiment would be located on the Inter-

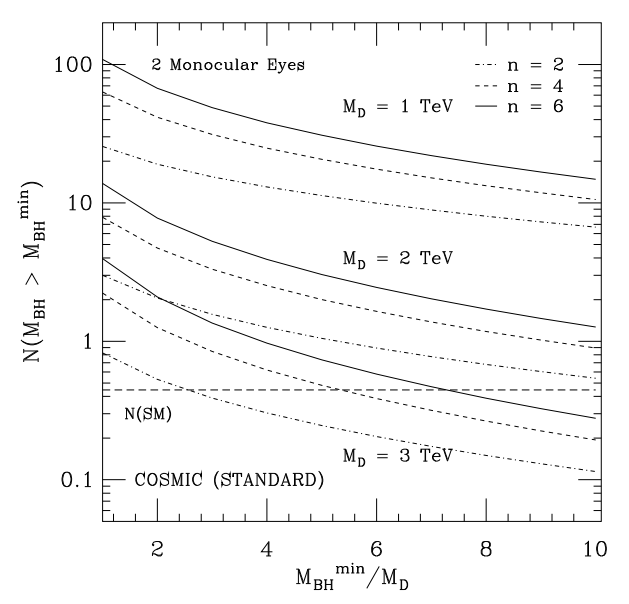

FIG. 8: The event rate per year for black hole production for 2 monocular eyes for OWL from the ESS cosmogenic electron neutrino flux (standard evolution), for $n=2,4,6$ extra dimensions, $M_{D}=1,2,3 \mathrm{TeV}$ as a function of $M_{\mathrm{BH}}^{\mathrm{min}} / M_{D}$. Also shown is the standard model event rate.

national Space Station, $380 \mathrm{~km}$ above the Earth. The conversion factor from the 2 telescopes to one at a reduced altitude amount to a reduction of the event rate by a factor of about 0.2 compared to OWL.

The threshold for OWL detection of horizontal air showers is on the order of $10^{10} \mathrm{GeV}$. By rescaling the effective aperture for OWL detection of electron neutrinos by the ratio of the standard model plus black hole cross section to the standard model neutrino-nucleon cross section, one can arrive at event rate predictions given the ESS incident electron neutrino flux. The range of electron neutrino induced event rates goes from 100 events per year for $n=6$ and $M_{D}=M_{\mathrm{BH}}^{\min }=1 \mathrm{TeV}$ to less than one event per year for $M_{D}=3 \mathrm{TeV}$ and $M_{\mathrm{BH}}^{\min }=10 \cdot M_{D}$ for the standard evolution of the cosmogenic flux as shown in Fig. 8. The standard model event rate is about 0.5 events/year. The strong evolution of the cosmogenic flux gives a factor of 2 enhancement for both signal and background. The OWL rates are about a factor of 20 times larger than the IceCube downward contained event rates for an energy threshold of $10^{8} \mathrm{GeV}$. IceCube will optimally detect fluxes with large low energy components, e.g., $F_{\nu} \sim 1 / E^{2}[27]$.

If all three neutrino flavors are included in the event rate calculation, the standard model rates increase by a factor of about 2 , while the black hole rates are increased by a factor of 3 since the black hole cross section is flavorblind. (The standard model rate depends on how much of the incident neutrino energy is translated to the shower in the final state.) Including these factors, one finds that OWL would be able to probe the fundamental Planck scale up to $3 \mathrm{TeV}$ for $n \geq 4$ even with one year of data taking. 


\section{FINAL REMARKS}

Described above are two ways to search for nonstandard model physics with ultrahigh energy neutrinos: searching for evidence of $\nu_{\mu} \rightarrow \nu_{\tau}$ oscillations with tau appearance in upward muon and upward shower rates, and mini-black hole production in neutrino induced horizontal air showers. Nonstandard model physics may be manifest in the flux of neutrinos, through exotic particle decay to neutrinos [4]. A wide range of experiments: underground Cherenkov detectors, surface arrays and satellite light collectors cover a huge range of energies from $\mathrm{GeV}$ to $10^{12} \mathrm{GeV}$ incident neutrinos. The view from the ultrahigh energy neutrino window on astrophysics and particle physics should provide interesting tests of the standard model and its extensions.

\section{Acknowledgments}

The work of S.I.D. has been supported in part by National Science Foundation Grant No. 0070998. The work of I.S. has been supported in part by the DOE under contracts No. DE-FG02-95ER40906 and DE-FG0293ER40792. The work of M.H.R. has been supported in part by the DOE under contract No. FG02-91ER40664.
[1] K. Greisen, Phys. Rev. Lett. 16, 748 (1966); V. Berezinsky and G. Zatsepin, in Proceedings of the $1976 \mathrm{DU}$ MAND Summer Workshop, ed. A. Roberts and R. Donaldson (Fermilab, Batavia, Illinois, 1977) p. 215.

[2] C.T. Hill and D.N. Schramm, Phys. Rev. D 31, 564 (1985); S. Yoshida and M. Teshima, Prog. Theoret. Phys. 89, 833 (1993); R.J. Protheroe and P.A. Johnson, Astropart. Phys. 4, 253 (1996); 5, 215(E) (1996).

[3] R. Engel, D. Seckel and T. Stanev, Phys. Rev. D 64, 093010 (2001).

[4] Y. Fukuda et al. [Super-Kamiokande Collaboration], Phys. Rev. Lett. 81, 1562 (1998).

[5] Q.R. Ahmad et al. [SNO Collaboration], Phys. Rev. Lett. 89, 0111302 (2002).

[6] D.V. Ahluwalia, C.A. Oriz and G.Z. Adunas, hep-ph/0006092 (unpublished); H. Athar, M. Jezabek and O. Yasuda, Phys. Rev. D 62, 1033007 (2000); H. Athar, Astropart. Phys. 14, 217 (2000).

[7] See, e.g., URL http://amanda.berkeley.edu/

[8] See, e.g., URL http://www.uao.gr/nestor/

[9] See, e.g., URL http://antares.in2p3.fr/.

[10] See, e.g., URL http://pheno.physics.wisc.edu/icecube/

[11] F. Halzen and D. Saltzberg, Phys. Rev. Lett. 81, 4305 (1998).

[12] S. Iyer, M.H. Reno and I. Sarcevic, Phys. Rev. D 61, 053003 (2000); S. Iyer Dutta, M.H. Reno and I. Sarcevic, Phys. Rev. D 62, 123001 (2000); 64, 113015 (2001).

[13] F. Becattini and S. Bottai, Astropart. Phys. 15, 323 (2001).

[14] K. Kravchenko et al. [RICE Collaboration], astro-ph/0112372

[15] D. Zavrtanik [AUGER Collaboration], Nucl. Phys. Proc. Suppl. 85, 3244 (2000).

[16] J.F. Krizmanic et al. [OWL/AirWatch Collaboration], in Proceedings of the 26th International Cosmic Ray Conference (ICRC 99) (1999) Vol. 2, 388-391; http://lheawww.gsfc.nasa.gov/docs/gamcosray/hecr/ owl_new.html.

[17] O. Catalano, Nuovo Cim. 24C, 445 (2001).

[18] C. Tyler, A. V. Olinto and G. Sigl, Phys. Rev. D 63, 055001 (2001).

[19] L. A. Anchordoqui, J. L. Feng, H. Goldberg and A. D. Shapere, Phys. Rev. D 65, 124027 (2002); Phys. Rev. D 66, 036007 (2002).

[20] J.G. Learned and S. Pakvasa, Astropart. Phys. 3, 267
(1995).

[21] D. Fargion, hep-ph/0206010 (unpublished); in Proceedings of the 27th International Cosmic Ray Conference (2001), Vol. 2, p. 903; G.W.S. Hou and M.A. Huang, astro-ph/0204145 (unpublished).

[22] R. Emparan, G. T. Horowitz and R. C. Myers, Phys. Rev. Lett. 85, 499 (2000);

[23] R. Emparan, M. Masip and R. Rattazzi, Phys. Rev. D 65, 064023 (2002).

[24] S. Nussinov and R. Shrock, Phys. Rev. D 64, 047702 (2001); Phys. Rev. D 59, 105002 (1999).

[25] J. L. Feng and A. D. Shapere, Phys. Rev. Lett. 88, 021303 (2002); L. Anchordoqui and H. Goldberg, Phys. Rev. D 65, 047502 (2002); Y. Uehara, Prog. Theor. Phys. 107, 621 (2002).

[26] A. Ringwald and H. Tu, Phys. Lett. B 525, 135 (2002); M. Kowalski, A. Ringwald and H. Tu, Phys. Lett. B529, 1 (2002).

[27] J. Alvarez-Muñiz, J.L. Feng, F. Halzen, T. Han and D. Hooper, Phys. Rev. D 65, 124015 (2002).

[28] P. Jain, S. Kar, D.W. McKay, S. Panda and J.P. Ralston, Phys. Rev. D 66, 065018 (2002).

[29] I. Antoniadis, Phys. Lett. B 246, 377 (1990); N. ArkaniHamed, S. Dimopoulos and G. R. Dvali, Phys. Lett. B 429, 263 (1998); I. Antoniadis, N. Arkani-Hamed, S. Dimopoulos and G. R. Dvali, Phys. Lett. B 436, 257 (1998); L.J. Randall and R. Sundrum, Phys. Rev. Lett. 83, 3370 (1999).

[30] See, for example, C. Quigg, Gauge Theories of the Strong, Weak, and Electromagnetic Interactions (Benjamin/Cummings, Menlo Park, 1983).

[31] R. Gandhi, C. Quigg, M.H. Reno and I. Sarcevic, Phys. Rev. D 58, 093009 (1998); Astropart. Phys. 5, 81 (1996).

[32] Yu. M. Andreev, V.S. Berezinsky and A. Yu. Smirnov, Phys. Lett. 84B, 247 (1979); V.S. Berezinsky and A.Z. Gazizov, Sov. J. Nucl. Phys. 29, 816 (1979).

[33] C. Adloff et al. [H1 Collaboration], Eur. Phys. J. C21, 33 (2001); J. Breitweg et al. [ZEUS Collaboration], Phys. Lett. B487, 53 (2000).

[34] L. Babukhadia [D0 Collaboration], hep-ex/0106069

[35] R.K. Ellis, Z. Kunszt, and E.M. Levin, Nucl. Phys. B420, 517 (1994) [Erratum-ibid. B433, 498 (1994)].

[36] M. Glück, S. Kretzer and E. Reya, Astropart. Phys. 11, 327 (1999).

[37] J. Kwiecinski, A.D. Martin and A.M. Stasto, Phys. Rev. 
D 59, 023002 (1999).

[38] M. H. Reno, I. Sarcevic, G. Sterman, M. Stratmann and W. Vogelsang, in Proceedings of Snowmass 2001, ed. N. Graf (eConf C010630, SLAC-R-599, 2001) P508.

[39] S. Kretzer and M.H. Reno, Phys. Rev. D 66, 113007 (2002).

[40] S. Iyer Dutta, M.H. Reno, I. Sarcevic and D. Seckel, Phys. Rev. D 63, 094020 (2001).

[41] J. Beacom, P. Crotty and E.W. Kolb, Phys. Rev. D 66, 021302 (2002).

[42] S. Iyer Dutta, M.H. Reno and I. Sarcevic, Phys. Rev. D 66, 077302 (2002).

[43] S. Iyer Dutta, M. H. Reno and I. Sarcevic, Phys. Rev. D 66, 033002 (2002).

[44] S. B. Giddings and S. Thomas, Phys. Rev. D 65, 056010
(2002); S. Dimopoulos and G. Landsberg, Phys. Rev. Lett. 87, 161602 (2001); T. G. Rizzo, in Proc. of the APS/DPF/DPB Summer Study on the Future of Particle Physics (Snowmass 2001) ed. R. Davidson and C. Quigg, hep-ph/0111230 K. Cheung, Phys. Rev. D 66, 036007 (2002).

[45] C.D. Hoyle, U. Schmidt, B.R. Heckel, E.G. Adelberger, J.H. Gundlach, D.J. Kapner and H.E. Swanson, Phys. Rev. Lett. 86, 1418 (2001).

[46] M. Acciarri et al. [L3 Collaboration], Phys. Lett. B 464, 135 (1999); Phys. Lett. B 470, 281 (1999).

[47] See, for example, P. Bhattacharjee and G. Sigl, Phys. Rept. 327, 109 (2000) and references therein. 